\title{
Complete suppression of torque ripple in BLDC machines caused by back emf and dead time
}

\author{
Miodrag Joksimovic, Student Member, IEEE, Emil Levi, Fellow, IEEE, Slobodan Vukosavic, Senior Member, IEEE
}

\begin{abstract}
This paper introduces a novel algorithm for suppresion of phase current harmonics in three-phase brushless dc motors caused by non-ideal back-emf waveform and dead-time effects. Proposed feedback acquisition chain obtains an exact information on all the relevant harmonics within each period of the fundamental. Design of the harmonic regulator based on the internal model control principle is given. The paper outlines the relevant details of implementation and the results of verification performed by both computer simulations and experimentally, using a laboratory prototype machine. Experimental results conducted in presence of non-sinusoidal back-emf and with erroneous dead time compensation prove the ability of the proposed solution to remove the stator current harmonics quickly, in just two fundamental periods.
\end{abstract}

Index Terms-BLDC, adaptive control, harmonic compensation, dead time compensation.

\section{INTRODUCTION}

B RUSHLESS dc motors are extensively used in various industrial applications, ranging from appliance-grade to high-end applications [1], [2], [3]. Most machines with high specific torque have an inherently large torque ripple, which is a shortcoming that limits their use in high-bandwidth applications. The main electrical factor that causes the torque ripple in BLDC motors are current harmonics, which depend on control technique, dead time of the inverter, and emf harmonics [4].

Various attempts to remove the torque ripple in steady state and during transients of BLDC motors have been made. Solutions reported in [5] - [6] consider BLDC drives with commutation scheme where two out of three phases are conducting at any instant and they report reduction of the torque ripple down to $5 \%-8 \%$. A dc-link voltage modulation circuit to temporarily increase dc-link voltage in the current commutation period is introduced in [7], resulting in torque ripple of $7.75 \%$ at high, and $6 \%$ at low speeds.

Solutions reported in [8] and [6] propose modification of the dc-link circuit and reduce commutation-related torque ripple down to 5\%. A method presented in [9] uses a spider based controller, and reports a torque ripple of $7 \%$. In [10], the authors prove that the torque ripple could be considerably lower in BLDC motors with sinusoidal phase currents provided that the current waveforms are free from harmonic distortions. Most BLDC drives with sinusoidal currents and fast torque response adopt Field Oriented Control (FOC), which is commonly achieved with synchronous frame controllers having the current loop bandwidth $f_{b w}>1 \mathrm{kHz}$ [11]. In the presence of non-sinusoidal back-emf, most relevant harmonics are at

Manuscript received Month xx, 2xxx; revised Month xx, xxxx; accepted Month x, xxxx. frequencies well beyond the bandwidth frequency $f_{b w}$, and the current controller cannot suppress the stator current content at frequencies of such harmonics.

The combination of back-emf harmonics [12] and relatively low phase impedance gives rise to non-negligible amplitudes of undesired phase current harmonics, which cause acoustic noise and the torque ripple [1], [13]. If these harmonics remain unaccounted for, the drive is not suitable for applications with strict requirements on vibrations, torque ripple and noise.

This paper proposes a novel algorithm for suppression of higher harmonic currents, arising from both the back-emf and dead time, by means of a novel harmonic-suppression controller. Phase currents are sampled, processed in the prescribed manner, and passed on to harmonic regulators, each implemented in a corresponding frame of reference, which give voltage commands required to suppress current harmonics.

The paper is organised as follows. Proposed feedback acquisition chain which outputs the relevant stator current harmonics in each period of the fundamental is presented in section II. The structure and parameter setting of harmonic suppression controllers are given in section III. Simulation results are presented in section IV, with a subsequent full experimental verification provided in section V. Section VI provides the conclusions of this paper.

\section{Feedback AcQuisition Chain}

Efficient suppression of back-emf caused current harmonics requires their quick and accurate detection from the stator currents, which in turn calls for precise current measurement. Proposed approach relies on oversampling and PWM averaging [14], followed by extraction of individual harmonics by one-fundamental-period averaging in multiple reference frames [13].

\section{A. One-Fundamental-Period Averaging}

The amplitude and frequency of back-emf harmonics change with the rotor angular velocity. Compared to the time constant of a high-bandwidth current regulator, such changes are relatively slow. One can then assume that the current harmonics arising from the non-sinusoidal back-emf are almost constant during one period of the fundamental frequency. Without the loss of generality, it can be assumed that the phase currents $I_{a b c}$ have the fundamental, the $5^{\text {th }}$ and the $7^{\text {th }}$ harmonic components:

$$
\begin{aligned}
I_{a} & =A_{1} \sin \left(\omega_{e} t+\phi_{1}\right)+A_{5} \sin \left(-5 \omega_{e} t+\phi_{5}\right)+ \\
& +A_{7} \sin \left(7 \omega_{e} t+\phi_{7}\right) .
\end{aligned}
$$

The minus sign in the fifth harmonic indicates negative sequence. Transformation to the synchronous reference frame 


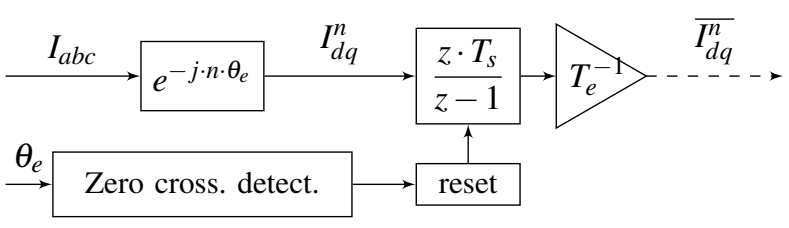

Fig. 1: Principle of measuring the $n^{\text {th }}$ current harmonic.

requires Clarke's and Park's transformation with $\theta_{e}=+\omega_{e} t$ and provides $I_{d q}$ currents. Using complex notation, $I_{d q}$ is $I_{d q}=I_{d}+j \cdot I_{q}=A_{1} e^{j \phi_{1}}+A_{5} e^{j\left(-6 \omega_{e} t+\phi_{5}\right)}+A_{7} e^{j\left(6 \omega_{e} t+\phi_{7}\right)}$,

where $\mathrm{A}_{1}, \mathrm{~A}_{5}, \mathrm{~A}_{7}$ and $\phi_{1}, \phi_{5}, \phi_{7}$ are amplitudes and phases of the first, the fifth and the seventh harmonic components, respectively, and $\omega_{e}$ is the fundamental electrical angular frequency of the machine. Applying Park's transformation with $\theta_{6 e}=-6 \omega_{e} t$ on the $I_{d q}$, one would acquire the currents in the $5^{\text {th }}$ harmonic synchronous frame

$$
\begin{aligned}
I_{d q}^{5} & =I_{d q} \cdot e^{j 6 \omega_{e} t} \\
& =A_{1} e^{j\left(6 \omega_{e} t+\phi_{1}\right)}+A_{5} e^{j \phi_{5}}+A_{7} e^{j\left(12 \omega_{e} t+\phi_{7}\right)} .
\end{aligned}
$$

The $\mathrm{A}_{5}$ component of the stator current (3) is obtained by considering the average value obtained within one period of the fundamental,

$$
\overline{I_{d q}^{5}}=\overline{I_{d}^{5}}+j \cdot \overline{I_{q}^{5}}=\frac{1}{T_{e}} \int_{0}^{T_{e}} I_{d q}^{5} d t=A_{5} \cdot e^{j \phi_{5}} .
$$

Assuming that the rotor speed does not change over one fundamental period, $d t$ is replaced by $d\left(\theta_{e} / \omega_{e}\right)$ to obtain

$$
\overline{I_{d q}^{5}}=\frac{1}{2 \pi} \int_{0}^{2 \pi} I_{d q}^{5} d \theta_{e}=A_{5} \cdot e^{j \phi_{5}} .
$$

Thus, if the current $I_{d q}^{5}$ is integrated and the integrator is reset at each zero-crossing of the angle $\theta_{e}$, the integrator output captured prior to the reset will provide the information on $\mathrm{A}_{5}$ and $\phi_{5}$. A block diagram of the described method is given in Fig. 1. Using the same reasoning, it is possible to extract the amplitude and phase of all the relevant harmonics.

\section{B. Suppressing the Impact of Drive Transients}

The presented method of current harmonic measurement relies on the fact that the integral of a sinusoidal function over its period is equal to zero. However, in practical implementations, the amplitude of the fundamental current constantly changes, since the flux and torque controllers permanently alter $I_{d}^{r e f}$ and $I_{q}^{r e f}$ current references, the latter being related to the output of the speed or position controller. Permanent changes of $I_{d q}^{r e f}$ affect the amplitude of fundamental currents, which affect the measurement of relevant harmonics. The worst case scenario would be the one in which the amplitude of the fundamental changes at one half of the fundamental period. In this case, the average value of the fundamental, seen from the harmonic frame of reference, would differ from zero, and would give false measurement of harmonic current. This "false measurement" would then excite the harmonic regulator which would generate unnecessary voltage commands in an attempt to suppress non-existent harmonics, thus impairing the torque response and the drive performance. This phenomenon has to be dealt with.

If the fundamental components were known in advance, it could be possible to remove their impact on the harmonic feedback. With the references $I_{d}^{r e f}$ and $I_{q}^{r e f}$ of the fundamental currents, the actual currents could be derived from known

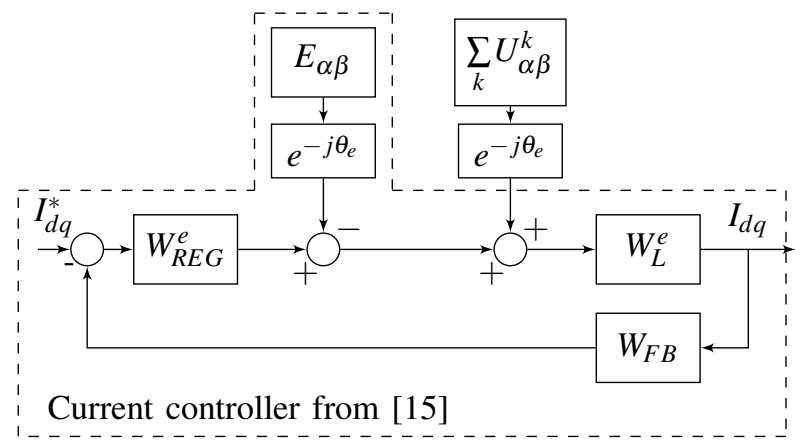

Fig. 2: Block diagram of fundamental current regulator, in the synchronous reference frame [15].

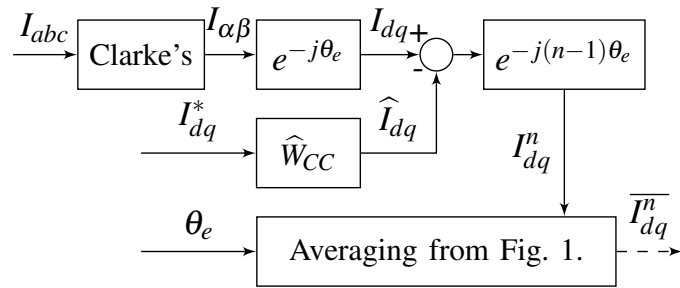

Fig. 3: Block diagram of harmonic acquisition path free from fundamental current transients.

transfer function of the digital current controller. The obtained estimates would correspond to the stator currents obtained in hypothetical case where the back-emf and dead-time disturbances are negligible. The closed loop transfer function of fundamental regulator is well known [15]. Looking at Fig. 2. and combining (6) and (13), one gets

$$
\begin{gathered}
W_{O L}^{e}=W_{R E G}^{e} \cdot W_{L}^{e}=\frac{\alpha_{d}}{z-1} \\
W_{C C}^{e}=\frac{I_{d q}}{I_{d q}^{r e f}}=\frac{W_{O L}^{e}}{1+W_{O L}^{e} \cdot W_{F B}}=\frac{2 \cdot \alpha_{d} \cdot z}{2 \cdot z^{2}+\left(\alpha_{d}-2\right) \cdot z+\alpha_{d}}
\end{gathered}
$$

Transfer function (7) can be used to create an estimator of undisturbed $I_{d q}$ currents

$$
\widehat{I}_{d q}=\widehat{W}_{C C}^{e} \cdot I_{d q}^{r e f} .
$$

Estimated values of $\widehat{I}_{d q}$ currents are subtracted from measured $I_{d q}$ currents to create a signal free from $I_{d q}$ transients, comprising only current components arising from higher harmonics. This signal is transformed into corresponding reference frame and averaged over one fundamental period, as shown in Fig. 3. Number $n$ in the second Park's transformation in Fig. 3 has an algebraic value corresponding to the relevant harmonic's order and sequence (positive or negative).

\section{HARMONIC COMPENSATION}

With the prescribed feedback acquisition chain, the sampling frequency is inherently set to the frequency of the fundamental. In practice, it is variable and it can not exceed a couple of hundreds of Hz. This sampling rate is considerably lower than the one used in conjunction with conventional digital current controllers. Therefore, the plant transfer function as seen by the harmonic suppression controller is considerably different from the plant transfer function seen by the conventional digital current controller. In order to design a harmonic controller, one must get an insight into the behaviour of current controlled machine enhanced by the proposed feedback acquisition chain, in order to derive the plant transfer function 
required for the proper design of the harmonic controller. After the individual harmonic controller's structure and parameters are obtained, the harmonic suppression system shall be obtained by parallel operation of $m$ harmonic controllers, as shown in Fig. 4.

\section{A. Fundamental Controller}

Harmonic controllers will be designed to work in conjunction with a fundamental current controller (FCC), which controls torque and flux of the machine, by controlling the direct and quadrature currents. This paper relies on the FCC presented in [14], with one-PWM-period feedback averaging and with advanced scheduling of control tasks. Transfer function of this regulator is [15]

$$
W_{R E G}(z)=\frac{U_{d q}}{I_{d q}}=\frac{\alpha_{d} L_{s}}{T_{S}} \cdot \frac{z \cdot e^{-j \omega_{e} T_{S}}-e^{-\beta}}{z-1},
$$

where $\alpha_{d}$ is an adjustable gain, and $T_{S}$ sampling period; $R_{S}$ and $L_{s}$ are phase resistance and inductance of the machine and $\beta=R_{s} \cdot T_{S} / L_{s}$. With $\alpha_{d}=0.20$, this regulator provides a fast response with overshoot of $0 \%$ and bandwidth $f_{b w}(-3 d b)=$ $0.0497 \cdot f_{s}, f_{s}=2 \cdot f_{p w m}$ being the sampling frequency, and exhibits stable behaviour with vector margin of 0.6917 . Block diagram of this FCC is given in Fig. 2.

In Fig. 2, the block diagram taken from [15] is delimited by the dashed-line box. Within this box, $E_{\alpha \beta}$ represents the electromotive force of the controlled motor, acting as the voltage disturbance. Sum of external voltage signals $U_{\alpha \beta}^{k}$, $k \in[5,7,11,13,17,19], k$ being the harmonic number of any relevant harmonic, is added to the voltage command $U_{\alpha \beta}$ in order to suppress the current harmonics. The signals $U_{\alpha \beta}^{k}$ are obtained from individual harmonic controllers.

Low-pass nature of the motor and reduced amplitudes of higher-order back-emf harmonics contribute to a significant reduction in amplitude of corresponding higher-order current harmonics. Therefore, it is reasonable to assume that the harmonics to be taken care of are the $5^{\text {th }}$ and $7^{\text {th }}$, accompanied in most cases with the $11^{\text {th }}$ and $13^{\text {th }}$, with rarely any need to consider higher order harmonics.

A brief example which illustrates how the order of higher emf harmonic affects the amplitude of higher current harmonic follows. From experiemntal recording, reported later in Section IV, the ratio of $17^{\text {th }}$ to $5^{\text {th }}$ harmonic amplitude $E_{17} / E_{5} \approx 1 / 3$ can be obtained. For a high enough fundamental frequency, the amplitude of currents arising from these harmonics will dominantly be limited by phase impedance of the machine $R_{s}+j \cdot \omega_{x} L_{s}, \omega_{x}=n \cdot \omega_{e}$ being the angular frequency of the given harmonic

$$
\frac{I_{17}}{I_{5}}=\frac{1 / \sqrt{R^{2}+\left(17 \cdot \omega_{e} \cdot L\right)^{2}}}{1 / \sqrt{R^{2}+\left(5 \cdot \omega_{e} \cdot L\right)^{2}}} \cdot \frac{E_{17}}{E_{5}} .
$$

At high enough angular frequency, phase resistance becomes an order of magnitude lower than the reactance $R<<X_{L}=$ $\omega_{e} \cdot L_{s}$. Approximating (10) gives

$$
\frac{I_{17}}{I_{5}}=\frac{\sqrt{\left(5 \cdot \omega_{e} \cdot L\right)^{2}}}{\sqrt{\left(17 \cdot \omega_{e} \cdot L\right)^{2}}} \cdot \frac{E_{17}}{E_{5}}=\frac{5}{17} \cdot \frac{1}{3}=0.098 .
$$

\section{B. Transfer Function of Equivalent Load}

Design of the harmonic suppression controller starts with the plant transfer function, namely, the function that relates the stator current harmonics, as an output, to the back-emf harmonic disturbance as an input. Such transfer function is obtained from disturbance transfer function $Y^{e}(z)$ of [15], relating the voltage disturbance to the output current. With one-fundamental-period averaging in the feedback chain, the sampling time of harmonic controllers is equal to one period of the fundamental. With settling time of contemporary current controllers [11] several tens of times shorter, a transient caused by the harmonic controller output would settle rather quickly. Therefore, it is reasonable to consider the steady state values of disturbance transfer function $Y^{e}\left(j \omega_{e}\right)$.

The equivalent load transfer function will be found by analysing the disturbance transfer function of the closed loop system given in Fig. 2.

The transfer function of the BLDC machine [11] in the discrete time domain and synchronous frame of reference is

$$
W_{L}^{e}(z)=\frac{T_{S}}{L} \cdot \frac{1}{z \cdot e^{-j \omega_{e} T_{S}-e^{-\beta}}},
$$

and the transfer function of the controller is given in (9). With principles of one-PWM-period averaging of the feedback path, and the advanced scheduling of control tasks [14], [15], and assuming $f_{s}=f_{p w m}$, the feedback transfer function is

$$
W_{F B}(z)=\frac{z+1}{2 z} \text {. }
$$

The disturbance transfer function is

$$
Y^{e}(z)=\left.\frac{I_{d q}(z)}{-E^{e}(z)}\right|_{I_{d q}^{r e f}=0}=\frac{W_{L}^{e}}{1+W_{L}^{e} \cdot W_{R E G}^{e} \cdot W_{F B}} .
$$

Substituting (12), (13), (9) in (15), one gets

$$
\begin{gathered}
Y^{e}(z)=\frac{T_{S} \cdot e^{-j \omega_{e} T_{S}}}{L} \cdot \frac{z-1}{z^{2} \cdot p_{1}+z \cdot p_{2}-e^{-\beta}\left(\alpha_{d}-1\right)}, \\
p_{1}=e^{-j \omega_{e} T_{S}} \quad, \quad p_{2}=e^{-j \omega_{e} T_{S}}\left(\alpha_{d}-1\right)-e^{-\beta},
\end{gathered}
$$

which represents the disturbance transfer function of the closed loop system in fundamental synchronous frame of reference.

When considering the stationary frame voltage disturbance at the frequency $\omega_{x}$, and assuming that the synchronous $d-q$ frame revolves at the speed $\omega_{e}$, disturbance transfer function in stationary frame $Y^{s}$ can be obtained from $Y^{e}$ as

$$
\begin{aligned}
Y^{s}(z) & =Y^{s}\left(e^{j \omega_{x} T_{s}}\right)=Y^{e}\left(e^{j \omega_{x} T_{S}} \cdot e^{-j \omega_{e} T_{S}}\right) \\
& =Y^{e}\left(e^{j\left(\omega_{x}-\omega_{e}\right) T_{S}}\right)=A\left(\omega_{e}, \omega_{x}\right)+j \cdot B\left(\omega_{e}, \omega_{x}\right) .
\end{aligned}
$$

Combining (15) with (16), setting $\omega_{e}$ to $314 \mathrm{rad} / \mathrm{s}$ and evaluating over the frequency band $f_{x} \in[-1000,1000] \mathrm{Hz}$ disturbance transfer function, shown in Fig. 5, is obtained.

The stator current response to back-emf harmonics can be described by real part (A) and imaginary part (B) of the disturbance transfer function (16) for any disturbance frequency $\omega_{x}$. While the curves in Fig. 5 provide $\mathrm{A}\left(\omega_{x}\right)$ and $\mathrm{B}\left(\omega_{x}\right)$ for the fixed fundamental frequency $\omega_{e}$, the curves in Fig. 6 provide the change of $\mathrm{A}\left(\omega_{e}, n\right)$ and $\mathrm{B}\left(\omega_{e}, n\right)$ for $n^{\text {th }}$ harmonic and variable excitation frequency $\omega_{e}$. Transfer function of the equivalent load, $W_{E Q}^{n}$, for a $n^{\text {th }}$ harmonic regulator is

$$
W_{E Q}^{n}=\frac{\overline{I_{d q}^{n}}}{U_{d q}^{n}}=A\left(\omega_{e}, n\right)+j \cdot B\left(\omega_{e}, n\right),
$$

where $\mathrm{A}\left(\omega_{e}, n\right)$ and $\mathrm{B}\left(\omega_{e}, n\right)$ are coefficients obtained from (16) for a known electrical angular velocity, and for a chosen harmonic $n=5,7,11,13$ or higher. 


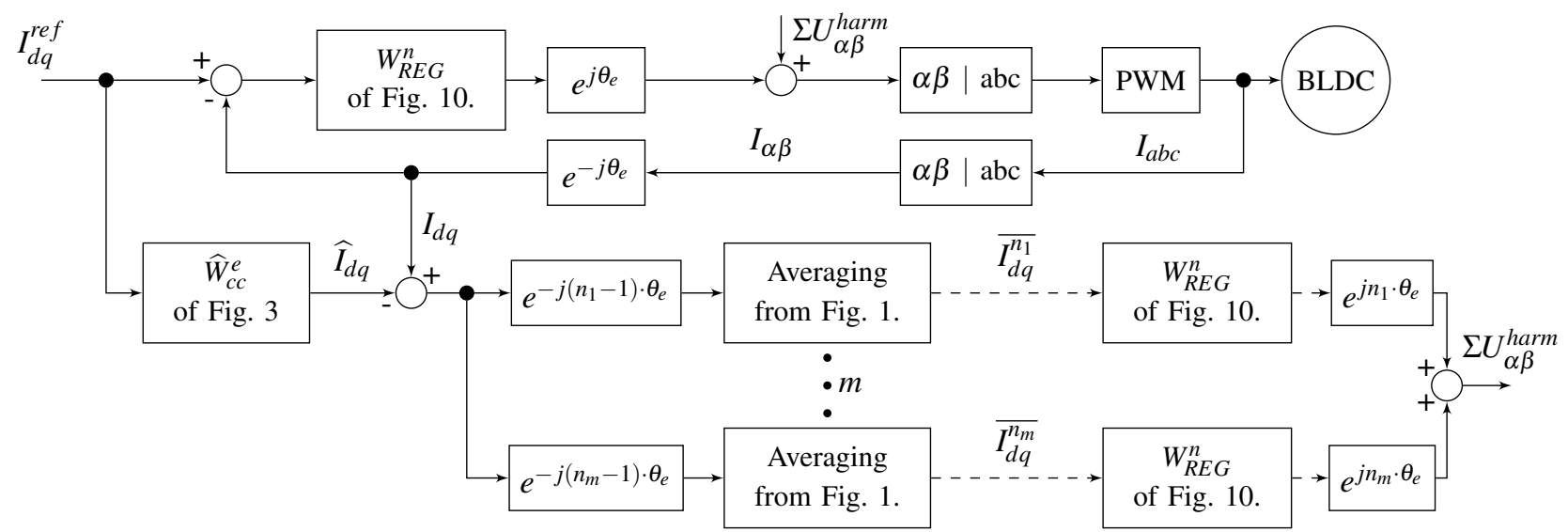

Fig. 4: Block diagram of the current controlled BLDC drive enhanced with harmonic controllers. Lower part of the diagram depicts $m$ harmonic controllers working simulatenously. Solid and dashed lines represent signals with sample rate $f_{s}$ and $f_{e}$ respectfully.

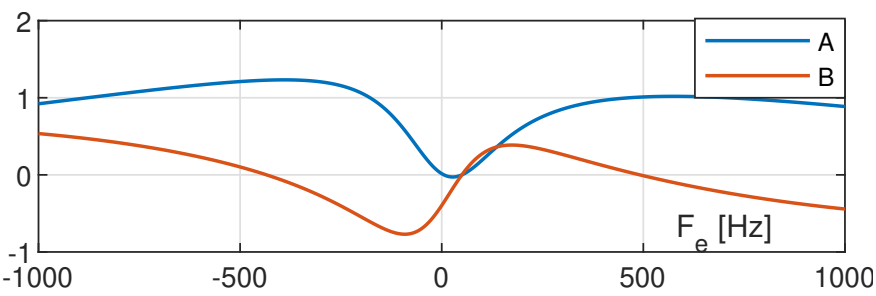

Fig. 5: Disturbance transfer function of closed loop system shown in Fig. 2. The results are obtained with electrical frequency of $100 \mathrm{~Hz}$

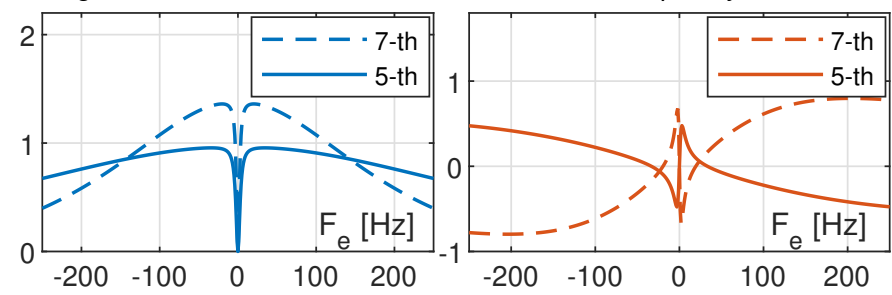

Fig. 6: Coefficients $A$ (left) and B (right) for selected harmonics, over a range of fundamental frequencies.

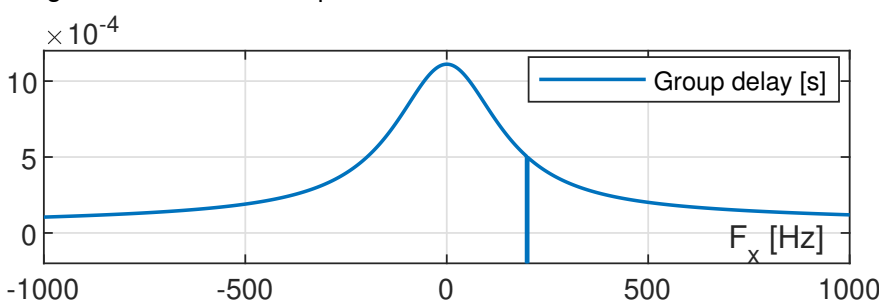

Fig. 7: Group delay of transfer function $Y^{s}$ from (15), for $\omega_{e}=314 \mathrm{rad} / \mathrm{s}$.

Relation (17) calculates the output current harmonic response $I_{d q}^{n}$ to the change of the voltage $U_{d q}^{n}$ as a static relation. This approach is based on the fact that the dominant time constant of the disturbance transfer function $Y^{e}(z)$ is considerably shorter than the fundamental period. To support this claim, a consideration of the group delay introduced by $Y^{e}(z)$ dynamics is made. Group delay of transfer function $Y^{e}(z)$, obtained with $\omega_{e}=50 \cdot 2 \cdot \pi$, is given in Fig. 7. Presumed step change of the $5^{\text {th }}$ harmonic of input voltage would result in harmonic current response delayed by $\tau=400 \mu$ s (Fig. 7). On these grounds, the group delay introduced by $Y^{e}(z)$ stands an order of magnitude lower than the sampling time of the harmonic controller up to the fundamental frequencies of $250 \mathrm{~Hz}$. If the fundamental frequency is larger, the impact of group delay becomes significant. The extent of such an impact is probed analytically and by means of computer simulations.

\section{Design of Harmonic Controller}

Each harmonic controller considers the feedback signals and derives the voltage command $U_{d q}^{n}$ which is added to the main voltage reference $U_{d q}$, Fig. 2. The structure of the harmonic controller has to be based on the properties of the load (16), (17). Adopting internal model approach [11], the controller is designed by inverting the transfer function of the load and multiplying the outcome by an integrator and an adjustable gain $\alpha_{n}$, where $n=5,7,11,13$ or higher, is the order of the considered harmonic:

$$
W_{R E G}^{n}(z)=W_{E Q}^{n}-1 \cdot \frac{\alpha_{n} \cdot z}{z-1}=\frac{A_{n}-j \cdot B_{n}}{A_{n}^{2}+B_{n}^{2}} \cdot \frac{\alpha_{n} \cdot z}{z-1},
$$

where $A_{n}=A\left(\omega_{e}, n\right)$ and $B_{n}=A\left(\omega_{e}, n\right)$ depend on the fundamental frequency, as shown in Fig. 6. Structure of harmonic controller is given in Fig. 8. Closed loop transfer function of the harmonic controller can be obtained by combining (18) and (17), and is given by

$$
W_{C L}^{n}(z)=\frac{W_{R E G}^{n}(z) \cdot W_{E Q}^{n}(z)}{1+W_{R E G}^{n}(z) \cdot W_{E Q}^{n}(z)}=\frac{z \cdot \alpha_{n}}{z \cdot\left(\alpha_{n}+1\right)-1} .
$$

Transfer function (19) has only one root at $z_{1}=\left(\alpha_{n}+1\right)^{-1}$, which for $\alpha_{n}>0$ is real and less than one. The correlation between s-domain and the z-domain poles is given by $z_{1}=$ $\exp \left(s_{1} / f_{e}\right)$. Aforementioned real z-domain pole corresponds to the real s-domain pole which resides on the negative part of the real axis between $-\infty$ and 0 . Parameter $\alpha_{n}$ will determine the settling time of the closed loop harmonic suppression controller. Closed loop step response of the harmonic regulator is given in Fig. 9.

Whenever $\omega_{e}$ changes, parameters $A_{n}$ and $B_{n}$ need to be updated. This can be done by creating a look-up table derived from (16), or by means of an appropriate polynomial approximation. The use of the same gain $\alpha_{n}$ over the range of rotor speeds ensures the same closed loop poles in $\mathrm{z}$ domain. Yet, the s-domain poles and the time response will depend on the sampling time. Since the sampling time is equal to one period of the fundamental, the response will be considerably slower at low speeds. This does not seem to present a problem since, at the same time, the amplitude 


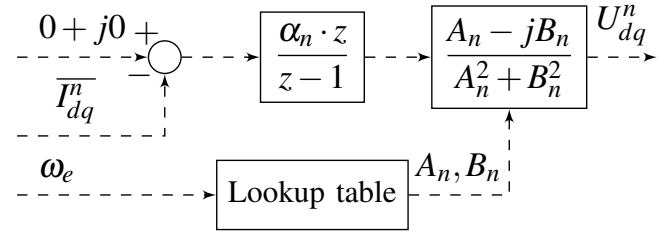

Fig. 8: The structure of $n^{\text {th }}$ the harmonic controller, for $n^{\text {th }}$ harmonic, $n \in[5,7,11,13]$ or higher.

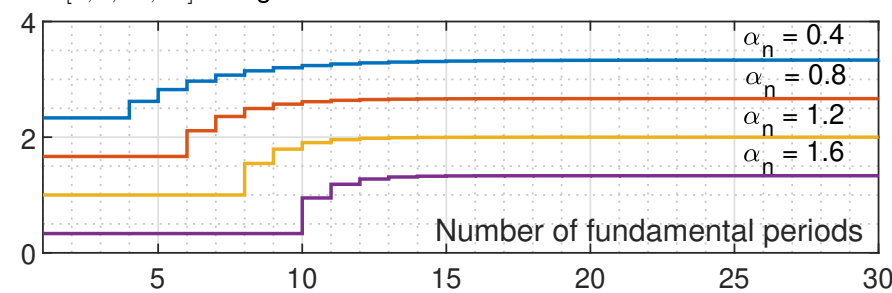

Fig. 9: Step response of the harmonic controller in closed loop, for $\alpha_{n} \in$ $[0.4,0.8,1.2,1.6]$.

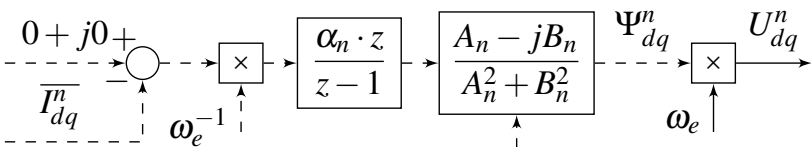

$$
\begin{aligned}
& \omega_{e} \rightarrow \text { Lookup table } \begin{array}{c}
A_{n}, B_{n} \\
-y_{-}
\end{array}
\end{aligned}
$$

Fig. 10: Extension of controller structure from Fig. 8 for speed independent operation.

of harmonic disturbances increases with the speed, and they remain low at low rotor speeds. Moreover, at low rotor speeds and low frequencies of said harmonic disturbances, they fold back within the bandwidth of the standard digital current controller [15], which is fully capable of eliminating their impact on the output current.

The outputs of the harmonic regulator are the voltages $U_{d}^{n}$ and $U_{q}^{n}$. At steady state, these voltages reach the values of corresponding back-emf harmonic disturbances. With backemf harmonics neutralized by the corrective action of corresponding harmonic controllers, the stator current harmonics are equal to zero. However, if a fast enough change of speed occurs, the amplitude of harmonic disturbances would change in proportion with the speed, introducing a difference between the actual outputs of harmonic controllers and newly established disturbances. Thus, the current harmonics will arise for a few fundamental periods, and get compensated by the corrective action of the controller. The settling time of these transients is determined by the parameter $\alpha_{n}$.

In order to eliminate the impact of sudden speed changes, controller structure of Fig. 8 can be further enhanced. In order to achieve speed-independent operation, harmonic controllers can be restructured to output the ratio between the voltage command $U_{d q}^{n}$ and the fundamental frequency $\omega_{e}$, the signal which has dimension of the flux and which is intrinsically speed-independent. In the end, prior to adding all the voltage corrections of individual harmonic controllers to the main voltage reference, the flux-like voltage corrections are multiplied with $\omega_{e}$ to get the voltage command. Corresponding change of the structure of the harmonic controller is depicted in Fig. 10.

\section{Dead-time Effects and their Compensation}

Dead time is inserted in switching signals to prevent the short circuit of the dc link. Even though this time is very short, it causes distortion of the voltage waveforms, differences between the voltage commands and output voltages, and introduces current harmonics [16], [17], [18]. The voltage error caused by the dead time can be modeled as [18]

$$
\Delta V_{a, b, c}=V_{d} \cdot \operatorname{sgn}\left(I_{a, b, c}\right),
$$

where $V_{d}$ represents the magnitude of the voltage error. Most implementations of the dead time are based on delaying the closure of the incoming switch by $T_{d}$, thus introducing an interval when none of the switches in one inverter leg conduct. The error magnitude $V_{d}$ is proportional to $T_{d}$. The deadtime effects are also temperature dependent and load-current dependent [17] and they are difficult to model and compensate.

From [18], voltage errors $\Delta V_{a}, \Delta V_{b}, \Delta V_{c}$ can be transformed from three-phase to the two-phase stationary frame. Corresponding voltage errors can be split into harmonic components,

$$
\left[\begin{array}{c}
\Delta V_{\alpha} \\
\Delta V_{\beta}
\end{array}\right]=V_{1} \cdot\left[\begin{array}{c}
\cos (\phi)+\frac{1}{5} \cos (5 \phi)-\frac{1}{7} \cos (7 \phi)-\ldots \\
\sin (\phi)-\frac{1}{5} \sin (5 \phi)-\frac{1}{7} \sin (7 \phi)+\ldots
\end{array}\right],
$$

where $V_{1}=4 \cdot V_{d} / \pi$ and $\phi=\omega_{e} \cdot t$ represents the phase of the current vector. It can be seen that the dead-time errors produce odd voltage harmonics. These harmonics impact the phase currents, and their effect can thus be measured using the one-fundamental-averaging presented in this paper. They can then be compensated with harmonic regulators. Namely, the harmonic controllers have the capability to suppress the backemf induced disturbances, and they can also remove stator current harmonics caused by the dead-time effects.

\section{E. Parameter Setting}

Transfer function of $n^{\text {th }}$ harmonic controller, from subsection III-C, is given in (18). Closed loop transfer function of the harmonic controller is

$$
W_{C C}^{n}=\frac{W_{R E G}^{n} \cdot W_{E Q}^{n}}{1+W_{R E G}^{n} \cdot W_{E Q}^{n}}=\frac{\alpha_{n} \cdot z}{\left(1+\alpha_{n}\right) z-1},
$$

where $\alpha_{d}$ is an adjustable parameter, which determines the closed loop response. For probed values of $\alpha_{n}$ ranging from 0.2 to 2 , the system is stable, and has no overshoots. For the purpose of the experiment, $\alpha_{n}$ is set to $\alpha_{n}=0.8$, resulting in the closed-loop settling time of 3 sampling periods.

\section{Simulation Results}

Proposed harmonic supressing system, depicted in Fig. 4, is first verified by computer simulations. Harmonic content of non-sinusoidal back-emf of BLDC motor is modelled according to actual measurements performed on a laboratory prototype, detailed in Appendix, running with fundamental frequency of $100 \mathrm{~Hz}$. Corresponding waveforms are given in Fig. 11. Simulation model includes BLDC motor modelled as R-L load with non-sinusoidal back-emf, PWM inverter with idealized power switches, and conventional digital current controller of [15]. In addition, the model is extended by the harmonic controller described in the previous section.

During simulations, $\mathrm{d}$-axis and q-axis current references of 
the drive are set to

$$
I_{d q}^{r e f}=4+j \cdot 0
$$

In operation with main digital current controller and without the harmonic controller, the waveforms of $\mathrm{d}$-axis and $\mathrm{q}$ axis currents are given in Fig. 12, with corresponding phase currents given in Fig. 13. It can bee seen from Fig. 13. that the conventional digital current controller of [15] does not suppress the current harmonics arising from the non-sinusoidal back-emf.

Before running the simulations of the whole system extended by the proposed harmonic controller, the structure and parameter setting of the harmonic controller have been verified, by checking the corresponding transient traces. With $\alpha_{n}=0.7$ and with the harmonic controller enabled at $t=0.56 \mathrm{~s}$, the waveforms (a-c) of Fig. 14 illustrate the suppression of the $5^{\text {th }}, 7^{\text {th }}$ and $11^{\text {th }}$ harmonics. The waveforms prove that, once enabled, harmonic controller manages to suppress harmonic currents in 2-3 sampling periods.

As discussed in subsection II-B, sudden change of drive references $I_{d}^{r e f}$ and $I_{q}^{r e f}$ can introduce error into harmonic feedback signals, obtained by averaging the stator currents in corresponding reference frames for each harmonic. These effects introduce errors into harmonic suppression structure and deteriorate the final outcome. In Fig. 15 the waveforms of simulated d-axis and q-axis currents are shown with the harmonic controllers enabled for $n \in[5,7,11,13,17]$ harmonics but without $\widehat{W}_{c c}^{e}(z)$ estimator introduced in Fig. 3. In these conditions, the step change of $I_{d}^{\text {ref }}$ introduced at $t=0.8 \mathrm{~s}$ produces considerable deterioration of the $I_{d}$ waveform. Simulation is repeated with $\widehat{W}_{c c}^{e}(z)$ estimator enabled, and with the harmonic feedback extraction organized in accordance with Fig. 3. Now, the waveforms of $I_{d}$ and $I_{q}$ in Fig. 15 (b) are not affected by the $I_{d q}$ steps, and the harmonic feedback acquisition proceeds undisturbed by the drive dynamics.

Effectiveness of the presented solution is verified by simulating the BLDC drive with digital current controller of [11], with proposed harmonic compensation and with the feedback acquisition system of Fig. 3. In Fig. 16, the drive runs at $\omega_{e}=628 \mathrm{rad} / \mathrm{s}$, and the plots show the stator currents $I_{a b c}$. Prior to $t=0.56 \mathrm{~s}$, harmonic controller is disabled, and the phase currents are distorted. At $t=0.56 \mathrm{~s}$, developed structure is enabled. In roughly two electrical periods, the impact of harmonic distortions is suppressed.

In order to verify the effectiveness of the controller extension of Fig. 10, suited to make the proposed suppressor robust against the changes in rotor speed and electrical frequency, the system is simulated while running at constant speed with the suppressor enabled and experiencing a sudden speed change. Corresponding waveforms of the stator direct and quadrature currents $I_{d q}$ are given in Fig. 17 along with the change in the rotor speed. Simulation results obtained in Fig. 17 demonstrate that the controller extension of Fig. 10 provides robustness against the speed changes and secures firm harmonic suppression even in drives with high acceleration rates.

\section{EXPERIMENTAL RESUlts}

Experimental verification is performed on a laboratory setup with a BLDC motor, hard-switched 3-phase PWM inverter and

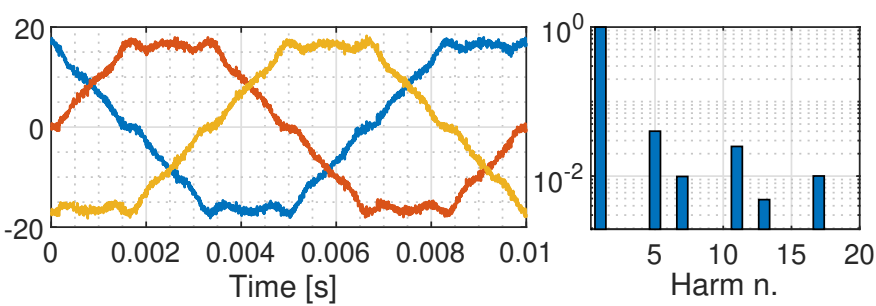

Fig. 11: Waveforms of back-emf obtained as the open-circuit voltages from the laboratory prototype at $f_{e}=100 \mathrm{~Hz}$ and corresponding spectrum.

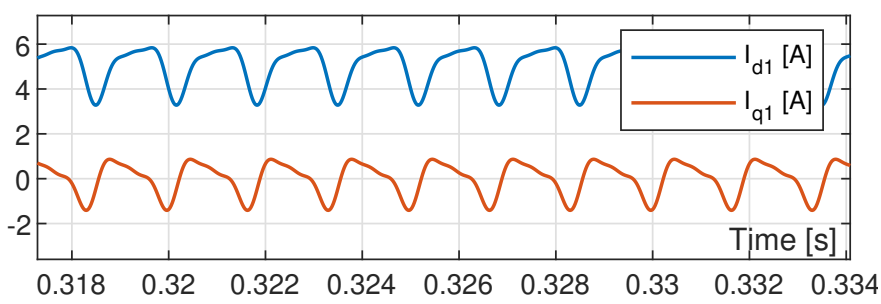

Fig. 12: Direct and quadrature current waveforms at electrical frequency of $100 \mathrm{~Hz}$.

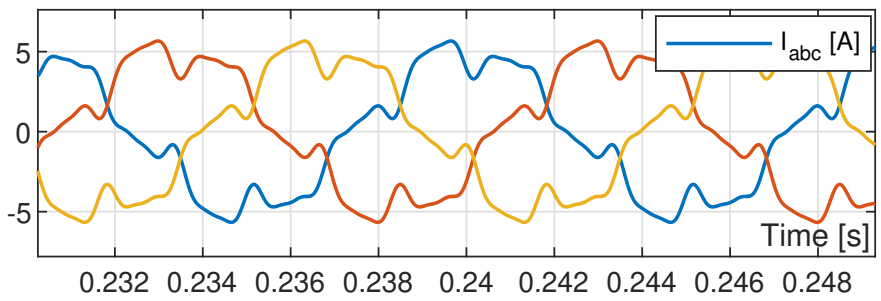

Fig. 13: Phase currents obtained from simulation, with $f_{e}=100 \mathrm{~Hz}$, depicting severe harmonic distortion.

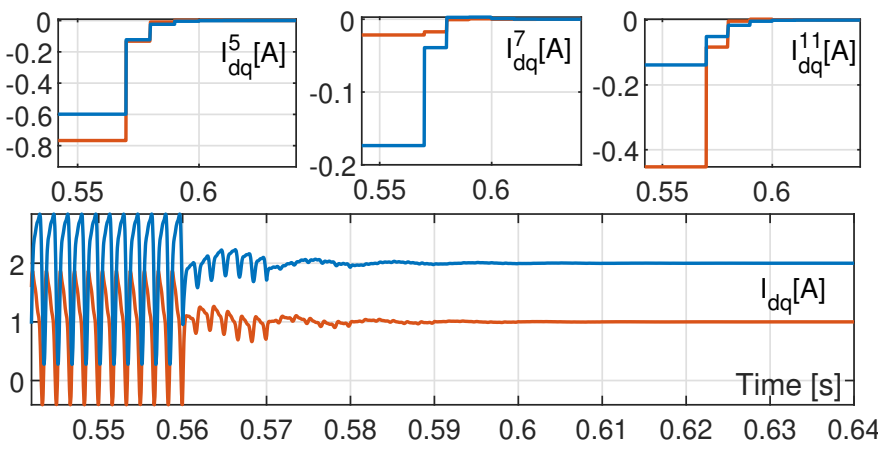

Fig. 14: Simulated waveforms of $I_{d q}$ currents, showing the dynamic characteristic of harmonic controllers. At $t=0.56 \mathrm{~s}$ harmonic controllers are enabled.

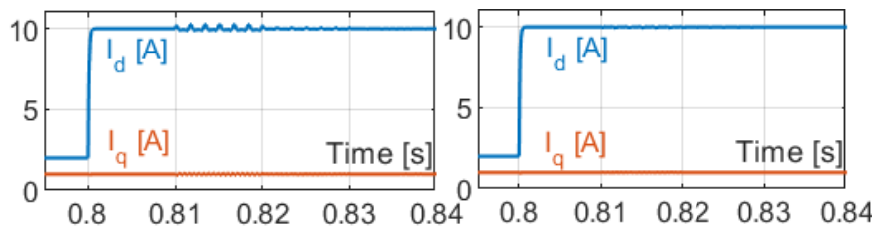

Fig. 15: With $\widehat{W}_{c c}^{e}$ of Direct and quadrature currents step response in situation where the harmonic acquisition is (a) affected and (b) unaffected by the drive dynamics.

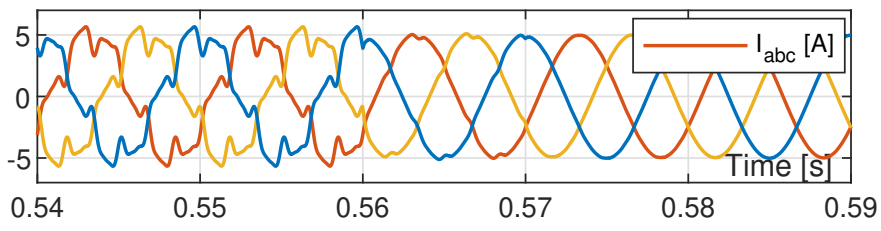

Fig. 16: Phase currents before and after harmonic compensation. Harmonic controllers are enabled at $t=0.56 \mathrm{~s}$. 


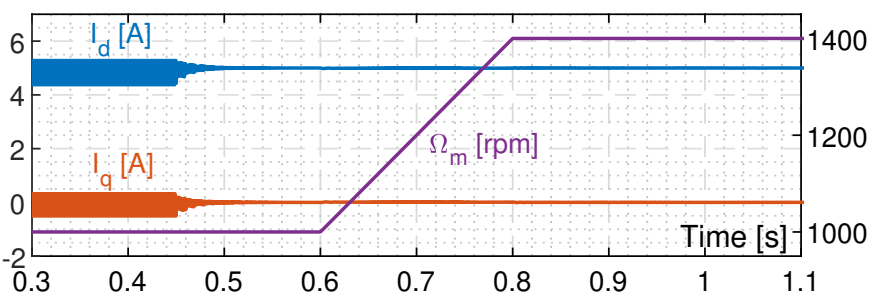

Fig. 17: The waveforms of direct and quadrature currents and the rotor speed obtained at the sudden speed ramp change. Harmonic controllers are enabled at $t=0.45 \mathrm{~s}$, and the machine experiences ramp speed change at $t=0.6 \mathrm{~s}$. Controller extension of Fig. 10 secures undisturbed operation with undistorted phase currents.

DSP-based programmable controller. The 8-pole BLDC motor has winding resistance of Rs $=0.1 \Omega$, inductance $L=100 \mu \mathrm{H}$, rated frequency of $200 \mathrm{~Hz}$ and rated current of $I_{\text {rated }}=25 \mathrm{~A}$. The machine is fed from a PWM inverter with $f_{p w m}=40 \mathrm{kHz}$, minimum dead time of $T_{d}=160 \mathrm{~ns}$, and with $V_{D C}=72 \mathrm{~V}$. The experimental setup has been developed for powering a small electrical vehicle. Experimental waveforms of non-sinusoidal back-emf obtained by measuring open-circuit voltages at $f_{e}=$ $100 \mathrm{~Hz}$ are given in Fig. 11.

The waveforms of Fig. 18 represent the phase current along with the corresponding spectrum obtained at the rotor speed of $\Omega_{\mathrm{meh}}=1500 \mathrm{rpm}$, where the fundamental frequency is $100 \mathrm{~Hz}$. The dead time is set to its rated value of $160 \mathrm{~ns}$ while the harmonic compensator is disabled. Due to the backemf harmonics and dead-time effects, the phase currents are distorted considerably. The waveforms of Fig. 19 represent the phase current along with the corresponding spectrum obtained at same speed of $n=1500 \mathrm{rpm}$ with the novel harmonic compensator fully enabled. Compared to Fig. 18, the relevant current harmonics are reduced more than 100 times. Residual value are mainly caused by the noise and quantization effects within the feedback chain. Total harmonic distortion is reduced from $25.25 \%$ down to $0.48 \%$. Results of Fig. 18 and Fig. 19 demonstrate the steady state operation of the system.

In order to check dynamic performance, Fig. 20 illustrates transient waveforms obtained upon turning on the harmonic compensator, which prove that the presented solution reduces the q-axis current ripple from 1.525A down to $0.211 \mathrm{~A}$, keeping the ripple below $0.8 \%$ of the rated current. Thus, reduction of the q-axis current ripple does not follow reported reduction of the total harmonic distortion (Fig. 19) and the residual q-axis current ripple comprises components that are not related to the back-emf harmonics and the dead time.

In order to prove that the step changes of the drive current references $I_{d q}^{r e f}$ do not disturb the harmonic suppressor, the $\mathrm{d}$-axis and q-axis currents are measured with the harmonic suppressor enabled and with the drive current references exhibiting step change. Experimental waveforms are given in Fig. 21. The traces of Fig. 21 (left) prove that the $I_{d q}^{r e f}$ step introduces disturbances into the feedback acquisition and results in visible disturbances of d-axis and q-axis currents. The undisturbed waveforms shown in Fig. 21 (right) correspond to the case where the current estimator is applied according to Fig. 3.

In order to verify the capability of the solution to suppress the harmonic currents caused by the dead time, the dead
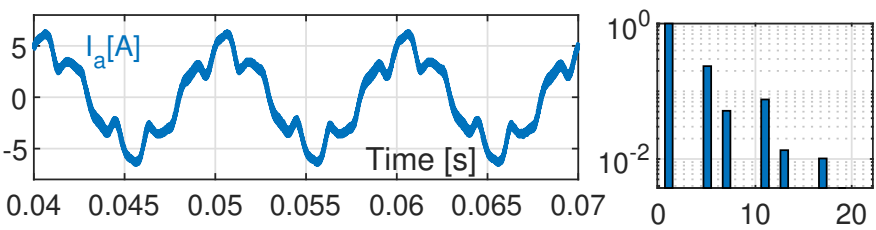

Fig. 18: The waveform and spectrum of the phase current obtained at $1500 \mathrm{rpm}$ without the novel harmonic suppressor.
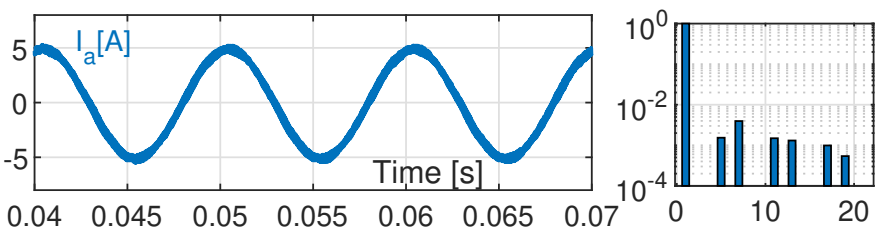

Fig. 19: The waveform and spectrum of the phase current obtained at $1500 \mathrm{rpm}$ with the proposed harmonic suppressor.
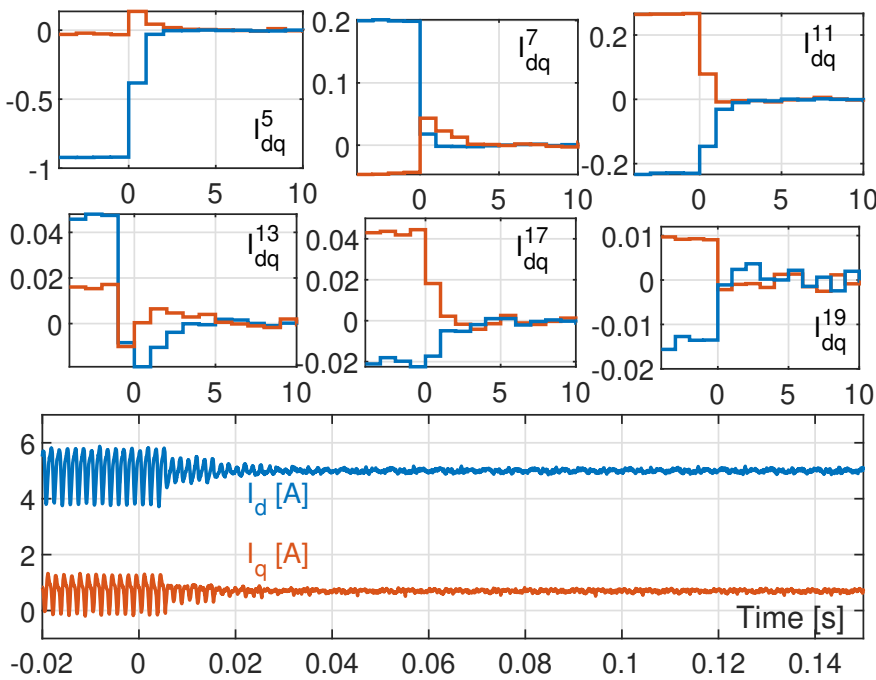

Fig. 20: Transient waveforms obtained upon turning on the proposed harmonic compensator. The waveforms are obtained at 1500rpm and with rated dead time of $160 \mathrm{~ns}$. The compensator is turned on at $\mathrm{t}=$ $0.005 \mathrm{~s}$. The plots (top left - center right) depict the harmonic feedback obtained in the corresponding reference frame for the six most relevant harmonics. The waveform (bottom) represents the d-axis and q-axis current components.
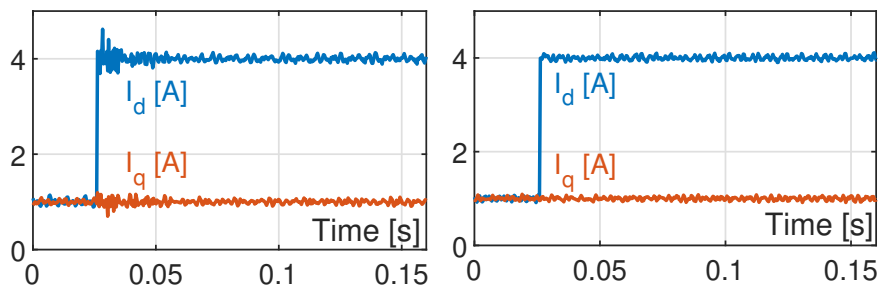

Fig. 21: Experimental waveforms of $d$-axis and q-axis currents obtained with $I_{d q}^{r e f}$ steps and with the proposed harmonic supressor enabled. Traces (left) are obtained without the estimator $W_{c c}(z)$ of Fig. 3, while traces (right) show that the use of the structure of Fig. 3 effectively removes corresponding disturbances.
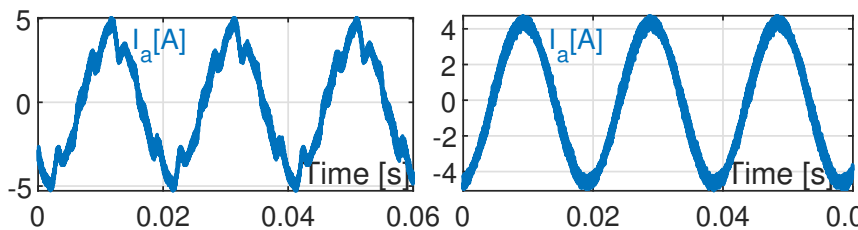

Fig. 22: Experimental waveforms of the phase currents obtained with an increased dead time of $5 \%$. The waveform (left) is obtained without the harmonic compensator. The waveform (right) is obtained with the developed harmonic compensator. 

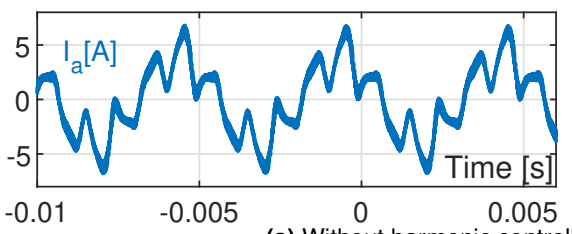

(a) Without harmonic controllers.
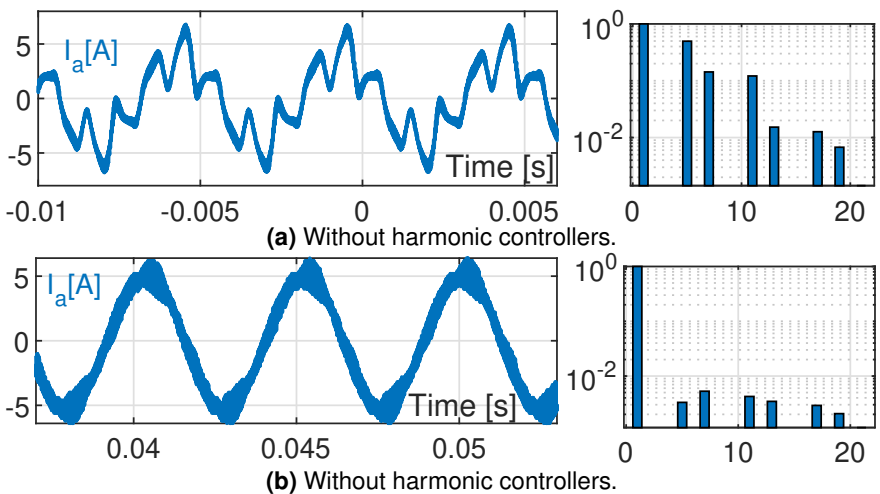

Fig. 23: Phase currents and respective harmonic content with and without harmonic controllers, at $f_{e}=200 \mathrm{~Hz}$.

time is intentionally increased to $5 \%$ of the PWM period. Corresponding waveforms of the phase currents are given in Fig. 22, without the harmonic suppressor (left) and with the harmonic suppressor (right).

Finally, the results of a test conducted at fundamental frequency of $200 \mathrm{~Hz}$ are shown in Fig. 23. At $200 \mathrm{~Hz}$, the THD without the harmonic suppressor reaches $53.11 \%$, while THD with the suppressor is below $0.91 \%$

\section{CONCLUSION}

Brushless permanent magnet dc motors are widely used both in high-volume applications such as electrical vehicles and in high-performance applications such as the industrial drives. The need to increase specific torque, reduce the volume and weight, and produce the motors with less active materials usually results in electrical machines with non-sinusoidal electromotive forces that give rise to current harmonics and the torque ripple.

Another source of the phase current harmonics and the torque ripple is the PWM inverter dead time. Whenever the drive runs at speeds commensurate to the rated speed, the frequency of corresponding phase current harmonics is well beyond the closed loop bandwidth of conventional digital current controllers and cannot be compensated.

In this paper, a novel harmonic suppressor is presented as an extension of the conventional digital current controller. Simulations and experimental results prove the method to be successful, both in steady state conditions and in transients. Whether running at high or low speeds, activation of the proposed harmonic controller effectively suppresses the stator current harmonics in just 2-3 periods of the fundamental waveform. Reduction of the stator current total harmonic distortion by more than 50 times is experimentally proved both at the rated speed and at one half of the rated speed. By considering the q-axis current waveforms, the torque ripple from all the sources is reduced more than 7 times and maintained below $1 \%$ even with the motor with considerable distortion of the back-electromotive force.

\section{APPENDIX - EXPERIMENTAL SETUP PARAMETERS}

\begin{tabular}{ll}
\hline \hline Motor type & Brushless DC \\
Number of poles & $2 \mathrm{p}=8$ \\
Motor rated current: & $\mathrm{I}_{\text {rated }}=24 \mathrm{~A}_{\text {rms }}$ \\
Rated electrical frequency: & $\mathrm{f}_{\text {rated }}=200 \mathrm{~Hz}$ \\
Back-EMF constant: & $\mathrm{k}_{\mathrm{e}}=0.0255 \mathrm{~V}_{\text {Peak }} /(\mathrm{rad} / \mathrm{s})$ \\
Stator resistance: & $\mathrm{R}_{\mathrm{s}}=0.1 \Omega$
\end{tabular}
DC-bus voltage: PWM frequency: Regulation frequency: Rated dead time: ADC resolution: Oversampling period: PWM method: DSP controller:
Stator inductance:

$$
\begin{aligned}
& \mathrm{L}_{\mathrm{s}}=\left(\mathrm{L}_{\mathrm{d}}+\mathrm{L}_{\mathrm{q}}\right) / 2=100 \mu \mathrm{H} \\
& \mathrm{V}_{\mathrm{DC}}=72 \mathrm{~V} \\
& \mathrm{f}_{\mathrm{PWM}}=1 / \mathrm{T}_{\mathrm{PWM}}=40 \mathrm{kHz} \\
& \mathrm{f}_{\mathrm{s}}=1 / \mathrm{T}_{\mathrm{s}}=20 \mathrm{kHz} \\
& \mathrm{T}_{\mathrm{d}}=160 \mathrm{~ns} \\
& \mathrm{~N}_{\mathrm{ADC}}=12 \mathrm{bit} \\
& \mathrm{T}_{\mathrm{ADC}}=\mathrm{T}_{\mathrm{PWM}} / 32 \\
& \text { Symmetrical PWM } \\
& \text { TMS320F28379D }
\end{aligned}
$$

\section{REFERENCES}

[1] T. Sheng, X. Wang, J. Zhang, and Z. Deng, "Torque-ripple mitigation for brushless dc machine drive system using one-cycle average torque control," IEEE Trans. Industrial Electronics, vol. 62, no. 4, pp. 2114-2122, 2015.

[2] Z. Q. Zhu and D. Howe, "Electrical machines and drives for electric, hybrid, and fuel cell vehicles," Proceedings of the IEEE, vol. 95, no. 4, pp. 746-765, 2007.

[3] C. Xia, G. Jiang, W. Chen, and T. Shi, "Switching-gain adaptation current control for brushless dc motors," IEEE Trans. Industrial Electronics, vol. 63, no. 4, pp. 2044-2052, 2016.

[4] M. Sumega, S. Zossak, P. Varecha, and P. Rafajdus, "Sources of torque ripple and their influence in bldc motor drives," Transportation Research Procedia, vol. 40, pp. 519 - 526, 2019.

[5] X. Li, G. Jiang, W. Chen, T. Shi, G. Zhang, and Q. Geng, "Commutation torque ripple suppression strategy of brushless dc motor considering back electromotive force variation," Energies, vol. 12, no. 10, p. 1932, 2019.

[6] W. Chen, Y. Liu, X. Li, T. Shi, and C. Xia, "A novel method of reducing commutation torque ripple for brushless dc motor based on cuk converter," IEEE Trans. Power Electronics, vol. 32, no. 7, pp. 5497-5508, 2017.

[7] R. K. Achary, S. Durgaprasanth, C. Nagamani, and G. S. Ilango, "A simple voltage modulator scheme for torque ripple minimization in a permanent magnet brushless dc motor," IEEE Trans. Power Electronics, vol. 35, no. 3, pp. 2809-2818, 2020.

[8] X. Li, C. Xia, Y. Cao, W. Chen, and T. Shi, "Commutation torque ripple reduction strategy of z-source inverter fed brushless dc motor," IEEE Trans. Power Electronics, vol. 31, no. 11, pp. 7677-7690, 2016.

[9] M. P. Maharajan and S. A. E. Xavier, "Design of speed control and reduction of torque ripple factor in bldc motor using spider based controller," IEEE Trans. Power Electronics, vol. 34, no. 8, 2019.

[10] S. Noguchi, K. Suzuki, and H. Dohmeki, "The efficiency comparison by exciting waveform of the square-wave brushless dc motor," in 18th Int. Conf. on Electrical Machines and Systems (ICEMS), 2015.

[11] S. N. Vukosavic, Grid-Side Converters Control and Design. Springer,international publishing, Switzerland, 2018.

[12] J. R. Hendershot and T. J. E. Miller, Design of brushless permanent-magnet motors. Magna Physics Pub; Clarendon Press Hillsboro, OH: Oxford, 2010.

[13] H. Dhulipati, S. Mukundan, C. Lai, K. Mukherjee, J. Tjong, and N. C. Kar, "Multiple reference frame-based extended concentrated wound pmsm model considering pm flux linkage and inductance harmonics," IEEE Trans. on Energy Conversion, vol. 34, no. 2, pp. 731-740, 2019.

[14] S. N. Vukosavic, L. S. Peric, and E. Levi, "Ac current controller with error-free feedback acquisition system," IEEE Transactions on Energy Conversion, vol. 31, no. 1, pp. 381-391, 2016.

[15] S. N. Vukosavic, L. S. Peric, and E. Levi, "A three-phase digital current controller with improved performance indices," IEEE Trans. Energy Conversion, vol. 32, no. 1, pp. 184-193, 2017.

[16] Y. Ji, Y. Yang, J. Zhou, H. Ding, X. Guo, and S. Padmanaban, "Control strategies of mitigating dead-time effect on power converters: An overview," Electronics, vol. 8, no. 2, p. 196, Feb 2019.

[17] Y. Yang, K. Zhou, H. Wang, and F. Blaabjerg, "Analysis and mitigation of dead-time harmonics in the single-phase full-bridge pwm converter with repetitive controllers," IEEE Trans. Industry Applications, vol. 54, no. 5, 2018.

[18] S. Kim and S. Park, "Compensation of dead-time effects based on adaptive harmonic filtering in the vector-controlled ac motor drives," IEEE Trans. Industrial Electronics, vol. 54, no. 3, 2007. 


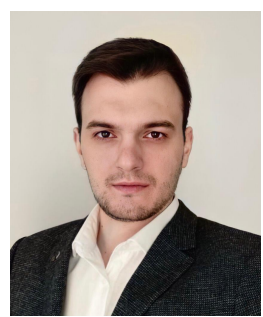

Miodrag G. Joksimovic (S'18) was born in Cetinje, Yugoslavia, in 1995. He received BSc and MSc degrees from the University of Belgrade, Belgrade, Serbia in 2019 and 2020, respectively, all in Electrical Engineering. In 2019 he was head of software development at H-Bridges, a first prized student team competing at IEEE International Future Energy Challenge. His research interests are focused on digital control of electrical drives and gridside converters, and power conversion in renewable energy sources.

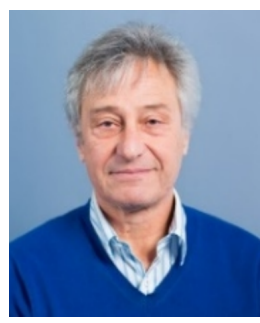

Emil Levi (S'89, M'92, SM'99, F'09) received his $\mathrm{MSc}$ and the $\mathrm{PhD}$ degrees in Electrical Engineering from the University of Belgrade, Yugoslavia in 1986 and 1990, respectively. He joined Liverpool John Moores University, UK in May 1992 and is since September 2000 Professor of Electric Machines and Drives. He served as a Co-Editor-in-Chief of the IEEE Trans. on Industrial Electronics in the 20092013 period. Currently he is Editor-in-Chief of the IEEE Trans. on Industrial Electronics and Editor-inChief of the IET Electric Power Applications. He is the recipient of the Cyril Veinott award of the IEEE Power and Energy Society for 2009 and the Best Paper award of the IEEE Trans. on Industrial Electronics for 2008. In 2014 he received the "Outstanding Achievement Award" from the European Power Electronics (EPE) Association and in 2018 the "Professor Istvan Nagy Award" from the Power Electronics and Motion Control (PEMC) Council.

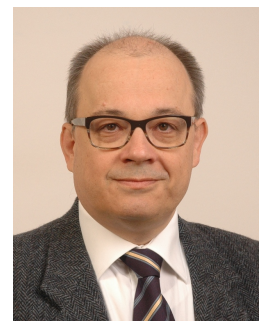

Slobodan N. Vukosavic (M'93, SM'12) was born in Sarajevo, Yugoslavia, in 1962. He received the B.S., M.S., and Ph.D. degrees from the University of Belgrade, Belgrade, Yugoslavia, in 1985, 1987, and 1989, respectively, all in Electrical Engineering. He was with the Nikola Tesla Institute, Belgrade, Yugoslavia, until 1988, when he joined the ESCD Laboratory of Emerson Electric, St. Louis, MO. Since 1991, he worked with Vickers Electric Company and MOOG Electric. S.N. Vukosavic is with the Department of Electrical Engineering, The University of Belgrade, and Serbian Academy of Sciences and Arts. His interests include digital control, power conversion in renewable energy sources and power quality. He has published over 100 papers, 4 textbooks and 7 monographs and has completed over 42 large R/D and industrial projects. 